Cuadernos de Investigación Geográfica. Tomo IX-1983 - págs. 29-40

(C) Colegio Universitario de La Rioja - Logroño

\title{
FALLAS CUATERNARIAS \\ EN LAS PROXIMIDADES DE ALCANADRE Y EN LA RIOJA BAJA
}

\author{
Ana Atarés del Campo* \\ Antonio Ortega-Lozano** \\ Félix Pérez-Lorente***
}

\begin{abstract}
RESUMEN
Los autores señalan la existencia de fallas cuaternarias dentro de la Depresión del Ebro, causantes de deformaciones en terrazas fluviales, hasta ahora atribuidas a movimientos halocinéticos.
\end{abstract}

\section{SUMMARY}

the authors point out the existence of quaternary faults in the Ebro Depression, causing alterations in fluvial terrasses, till now attributed to halocinetic movements.

Son varios los trabajos que citan las terrazas deformadas del río Ebro en La Rioja Baja, y todos ellos asocian tal deformación a movimientos halocinéticos de los yesos y sales terciarios. La existencia de fallas paralelas a estructuras y formas del relieve aparentes, así como la coincidencia entre las direcciones determinadas para las grandes fallas y las medidas de estrías en cantos impresos de las terrazas deformadas, nos hace pensar que es más acertado atribuir tales efectos a deformaciones cuaternarias relacionadas con fallas de desgarre sinistrosas de dirección N118E.

\footnotetext{
${ }^{*}$ Estación Meteorológica de Agoncillo. S.M.N. Logroño.

** Geo-Services. París.

*** Departamento de Geología. Colegio Universitario de La Rioja. Logroño.
} 
ANA ATARES DEL CAMPO, ANTONIO ORTEGA-LOZANO, FELIX PEREZ-LORENTE

\section{Localización geográfica (Fig. 1)}

Al Este de Logroño, siguiendo el curso del Ebro, sobre Navarra y La Rioja, hay una zona en la que se habían citado terrazas cuaternarias deformadas. Dedicamos nuestra atención principal a los alrededores de Alcanadre, 30TWM725952 en coordenadas U.T.M. sobre la hoja a escala 1:50.000 n 205 o bien 24-10. LODOSA.

\section{Localización geológica}

Los terrenos aflorantes son de edad terciaria y cuaternaria: los primeros, Miocenos, en y sobre los Yesos de los Arcos (RIBA, varios trabajos) y los segundos, de no menos de seis niveles de erosión y sedimentación (PEREZLORENTE, 1979).

\section{Estructuras observadas}

En el sector de la Depresión del Ebro a que nos referimos en esta nota hay una serie de hechos estructurales destacables que afectan a los terrenos cuaternarios, y que enunciados según la escala a la que se pueden observar son los siguientes:

- Depresión de Arnedo.

— Límites de las terrazas del Ebro, recientes y algunas antiguas.

- Pliegue del SE de Alcanadre.

- Termalismo de la línea Arnedillo-Fitero.

- Deslizamientos de ladera asociados con fallas.

- Fallas a escala de afloramiento.

- Terrazas cuaternarias plegadas.

- Cantos impresos.

- Cantos fracturados.

- Estrías sobre superficies de cantos y de fallas.

La Depresión de Arnedo (fig. 2 y 3), aunque estructuralmente es un domo o anticlinal complejo, es en suma una forma determinada por dos peculiaridades: una la variación de potencia de los Conglomerados de Yerga (ORTEGA LOZANO, et al., en prensa); y otra las fallas por las que está limitada. Por la parte norte, el sistema de fallas del Cidacos, al que sigue la Falla de Quel (que se puede prolongar al norte de Cabi Monteros) y que termina en falla inversa o en un sistema de fallas inversas; finalmente, el límite sur es la Falla de Arnedillo. 


\section{FALLAS CUATERNARIAS EN LA RIOJA BAJA}

Las terrazas de la Depresión del Ebro (foto 1; fig. 2) tienen terminaciones relativamente netas y paralelas. Esta dirección resalta al examinar tanto la cartografía de la figura 2 como la presentada en un trabajo de uno de nosotros (PEREZ-LORENTE, 1979). En los fotogramas de satélite son a veces especialmente claras tales direcciones, cuyo valor oscila entre N115-120E.

El pliegue anticlinal del SE de Alcanadre (fig. 2) es de tendencia angular, con el flanco sur vertical y el flanco norte de poco buzamiento; su eje buza suavemente hacia el este. Este anticlinal lo configuran los Yesos de Los Arcos. Su dirección es paralela a la ya mencionada, es decir N115-120E.

Existe una anomalía geotérmica comprobada, limitada por lo que conocemos, en las termas de Arnedillo y Fitero (Fig. 3). Esta anomalía, por otra parte muy interesante, se extiende entre ambos lugares mediante "una franja de unos 300 metros de anchura en la que la irradiación del calor desde el subsuelo crece con respecto a lo normal". (ALONSO, et al., 1980). La dirección de la anomalía es N118E.

El codo del Cidacos en la Depresión de Arnedo corresponde a la evolución del cauce de este río que se acopla al trazado de la Falla de Quel; portillos de Arnedo, de Quel y de Autol (PEREZ-LORENTE, 1979).

Los deslizamientos de ladera se hallan asociados en esta región, por lo general, con surgencias de agua y entornos con abundancia de vegetación. Entendemos que el agua, obligada a circular por superficies de falla, será la causante del aumento de plasticidad de las arcillas y margas terciarias, finalmente generadoras de tales deslizamientos.

A escala de afloramiento, cuando hay canteras suficientemente profundas para que desaparezcan los removilizados más recientes, se ven superficies de fallas con estrías horizontales en las que no hemos podido deducir el sentido de desplazamiento de los bloques.

De la misma forma que los deslizamientos asociados con las fallas están indicados en el mapa (fig. 2), las terrazas cuaternarias plegadas o basculadas también lo están. Hasta ahora hemos visto dos sinclinales al SW de Alcanadre, sobre la autopista del Ebro, y más al este, en la parte superior de un otero hay una terraza basculada; finalmente al norte de la autopista, en el nivel de dudosa posición, hay por lo menos dos afloramientos de terrazas que buzan hacia el sur (s.l.) entre 10 y 15 grados.

A menos escala siguen existiendo fenómenos debidos a los esfuerzos que afectan a esta región. Hay cantos cuya superficie tiene oquedades debidas a disolución (fotos 2 y 3): son los cantos impresos. Estas oquedades o impresiones se deben al efecto producido en la superficie de los cantos por otros más pequeños con los que se pone en contacto. Si el esfuerzo es perpendicular a la superficie del canto hay huecos con la misma dirección; si es tangente se producen estrías (foto 4) o acanaladuras; y si es oblícua, hay incisiones con una terminación brusca mientras que la terminación opuesta está formando un ángulo pequeño con la superficie del canto. 
No solamente se producen aquí cantos impresos debidos a la deformación, sino que también hay ejemplos en los que los cantos están fracturados. Este tipo de fracturas, según planos con deslizamiento, se encuentran silicificadas en algunos ejemplares.

Se han proyectado diversas medidas, en pequeño número dado que no se han podido tomar más datos fiables. Los polos correspondientes a los planos de fractura muestran una gran dispersión, así como los dos únicos datos de impresiones perpendiculares a las superficies de los cantos. Las estrías muestran con mayor claridad que el movimiento predominante es horizontal, y tienen un máximo pequeño en las proximidades de N120E. (Fig. 4).

\section{El sistema de fallas del Ebro}

Las estructuras mencionadas en el apartado anterior se corresponden con un sistema de fallas sinistrosas de desgarre. Puede ser que el anticlinal del SE de Alcanadre tenga una historia diferente, pero afecta a terrenos cuaternarios en el flanco sur por lo menos. No son fáciles de relacionar entre sí las diferentes estructuras observadas; por ejemplo: el basculamiento de la cuenca hacia el Este, acompañada por la migración de los lechos de los ríos, (PEREZLORENTE, 1979) con la falla inversa de Yerga, aunque estén en conexión con fallas N118E. Esto nos lleva a pensar que o bien no damos con la solución correcta o faltan datos para la interpretación o la historia de este sistema de fallas es algo más complicado de lo que a continuación se expone.

Las fallas de dirección N118E son sinistrosas y algunas de ellas por lo menos, activas en la actualidad (Falla de Arnedillo). Asociados con ellas hay cabalgamientos hacia el Este cuya magnitud de desplazamiento según la horizontal desconocemos, pero probablemente inferiores a $2 \mathrm{~km}$. (el mayor desplazamiento probable horizontal medido por nosotros en esta región.

\section{Conclusiones}

El Valle del Ebro en La Rioja Baja está determinado en gran parte por un sistema de fallas cuaternarias de dirección N118E. A este sistema de fallas corresponden, si no todos, algunos de los bordes de las sierras de la Demanda y Cameros.

Hay terrazas deformadas y límites de terrazas rectilíneos a gran escala por el movimiento de estas fallas.

Presenta un interés especial la anomalía geotérmica de la Falla de Arnedillo por cuanto pudiera utilizarse como fuente de energía.

Teóricamente, y a la espera de posteriores trabajos de investigación, parece que el esfuerzo compresivo principal causante de los movimientos cuaternarios es de dirección aproximada E-W. 


\section{BIBLIOGRAFIA}

ALONSO, I. y GOMEZ, J., 1980.- Estudio geológico-paleontológico del Jurásico del borde norte de la Sierra de la Demanda. Berceo, 99; 39-100, Inst. Est. Riojanos . Logroño.

CAPOTE, R., GUTIERREZ ELORZA, M., HERNANDEZ, A. y OLIVE, A., 1981.- Movimeintos recientes en la fosa del Jiloca (Cordillera Ibérica). V Reunión del Grupo Español de Trabajo del Cuaternario, pp. 245-257, Sevilla.

CASTIELlA, J., SOLE, J., VILLALOBOS, L. y DEL VALLE, J., 1977.- Mapa Geológico de España. E. 1:50.000. CALAHORRA. I.G.M.E., Madrid.

CRUSAFONT, M., TRUYOLS, J. y RIBA, O., 1966.- Contribución al estudio de la estratigrafía del terciario continental de Navarra y Rioja. Not. y Com. del I.G.M.E., 90; Madrid.

ORTEGA LOZANO, A. y PEREZ-LORENTE, F., 1983.- El terciario en la Depresión de Arnedo. Inst. Est. Riojanos (en prensa).

PEREZ-LORENTE, F., 1979.- Niveles de erosión y acumulación en La Rioja Central y Oriental. Cuad. Inv. (Geogr. Hist.), 5 (1-2): 37-50, Logroño.

RIBA, O., 1964.- Estructura sedimentaria del terciario continental de la Depresión del Ebro en su parte riojana y navarra. XX Congr. Geogr. Int. pp. 127-138, Zaragoza.

RIBA, O. y BOMER, B., 1957.- Les terrasses et glacis du bassin de l'Ebre dans la Ribera de Navarra et La Rioja Baja. Livr. Guide Exc. 3 INQUA. Congr. Int. Madrid-Barcelona. 7-10,París. 
ANA ATARES DEL CAMPO, ANTONIO ORTEGA-LOZANO, FELIX PEREZ-LORENTE

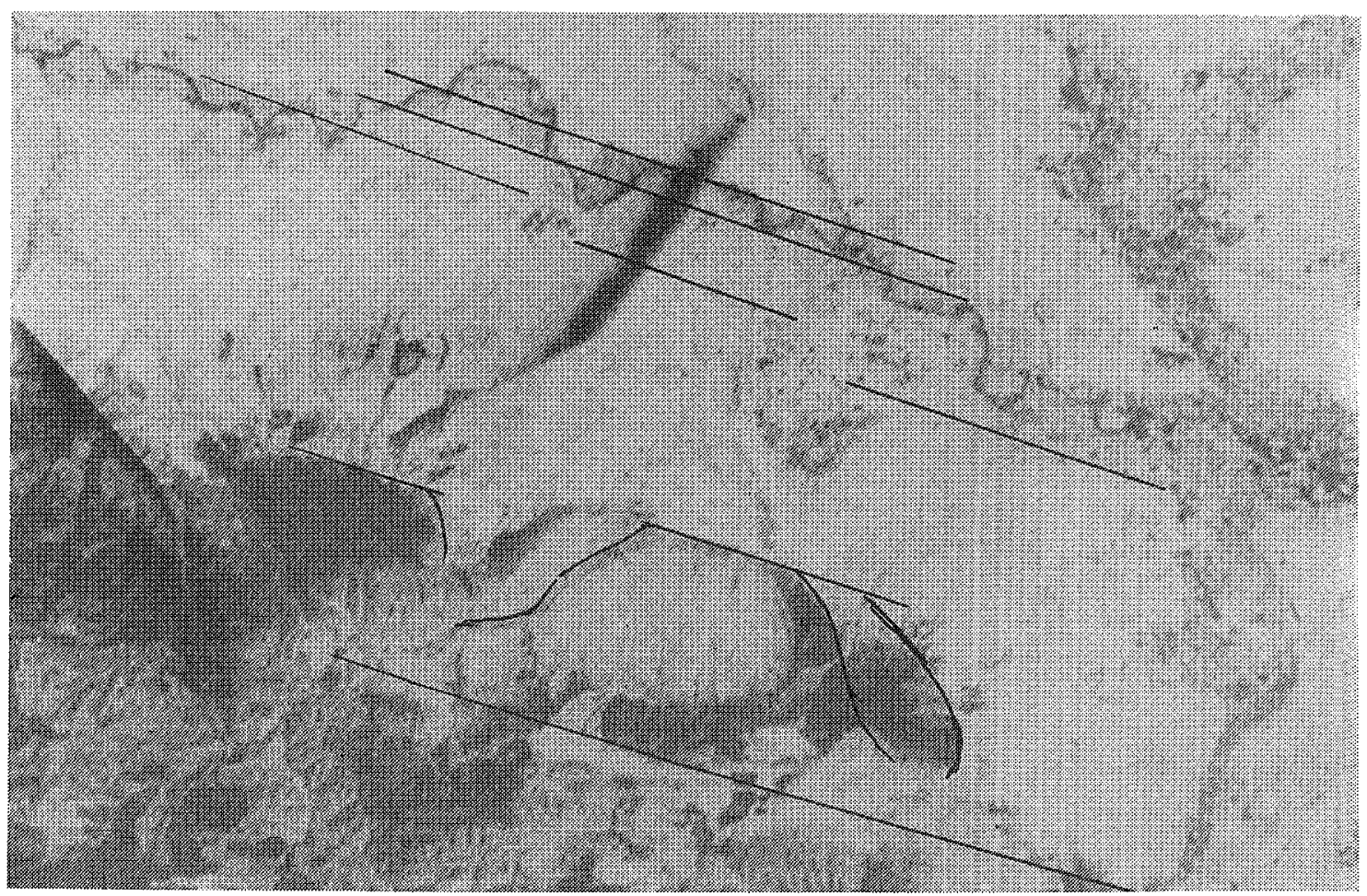

FOTO 1

Lineamientos importantes visibles en la Rioja Baja. Imagen de satélite.

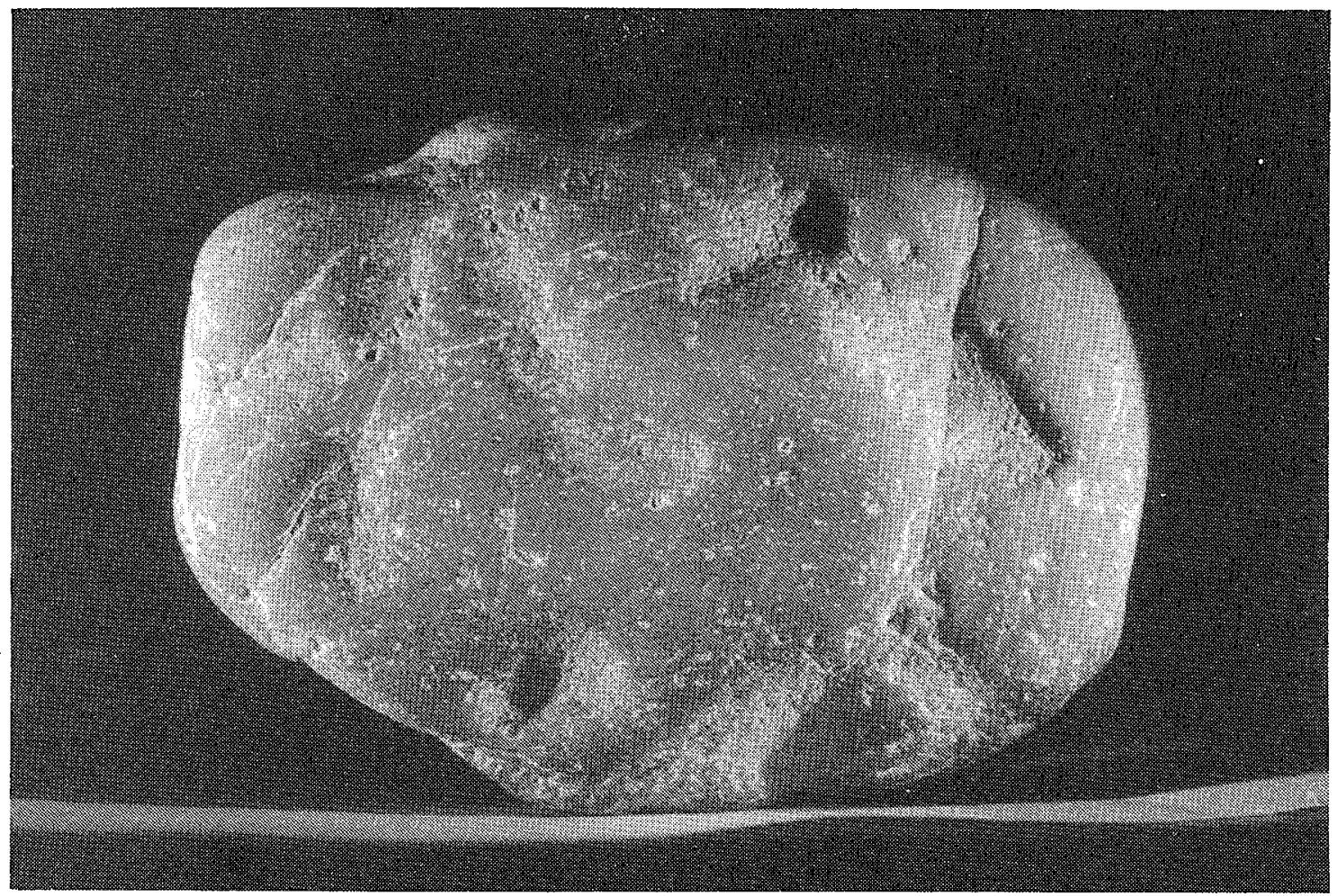

FOTO 2

Canto impreso. Recogido en nivel de dudosa posición. 
FALLAS CUATERNARIAS EN LA RIOJA BAJA

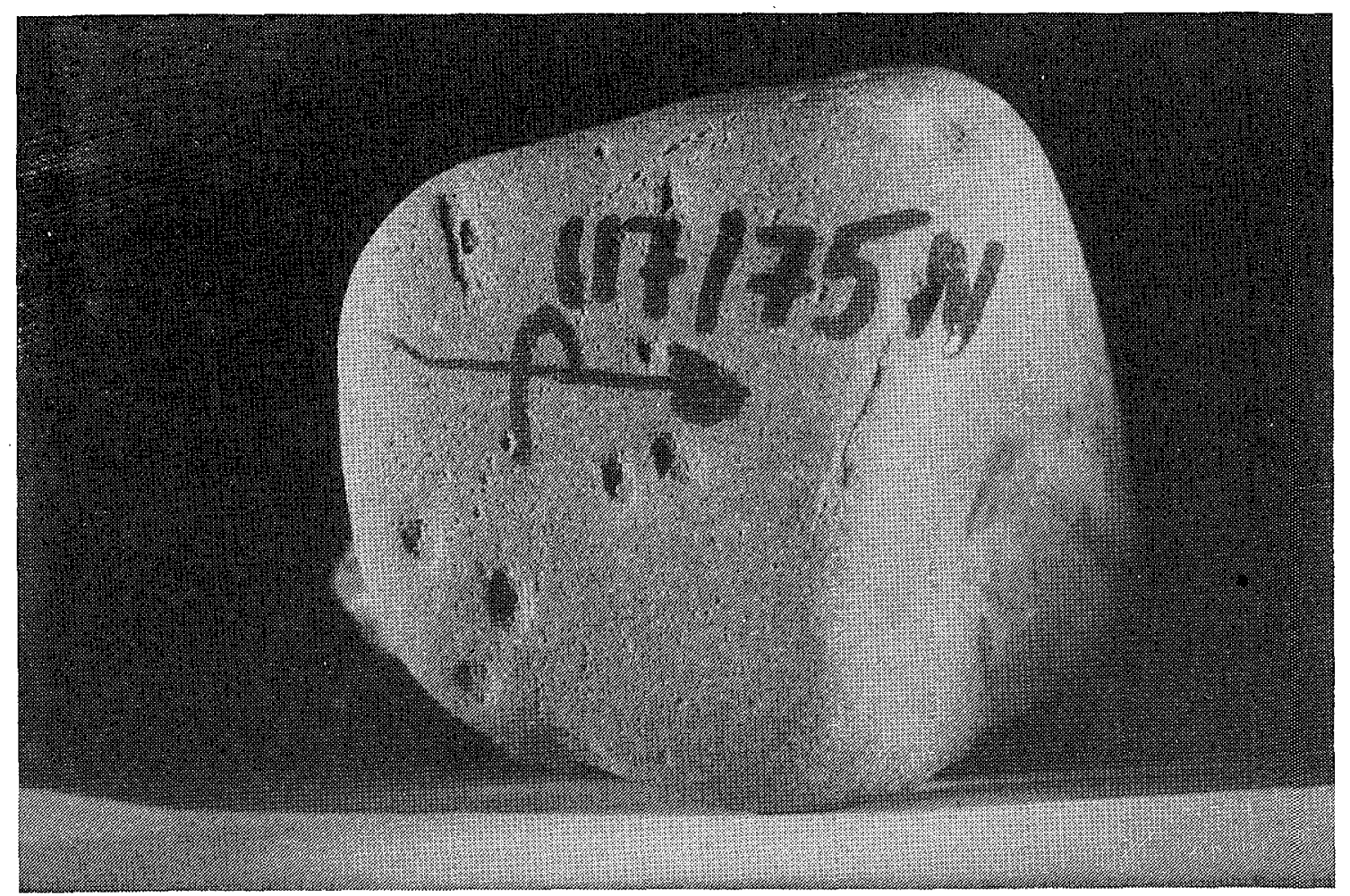

FOTO 3

Canto impreso recogido en la carretera de Alcanadre a Lodosa.

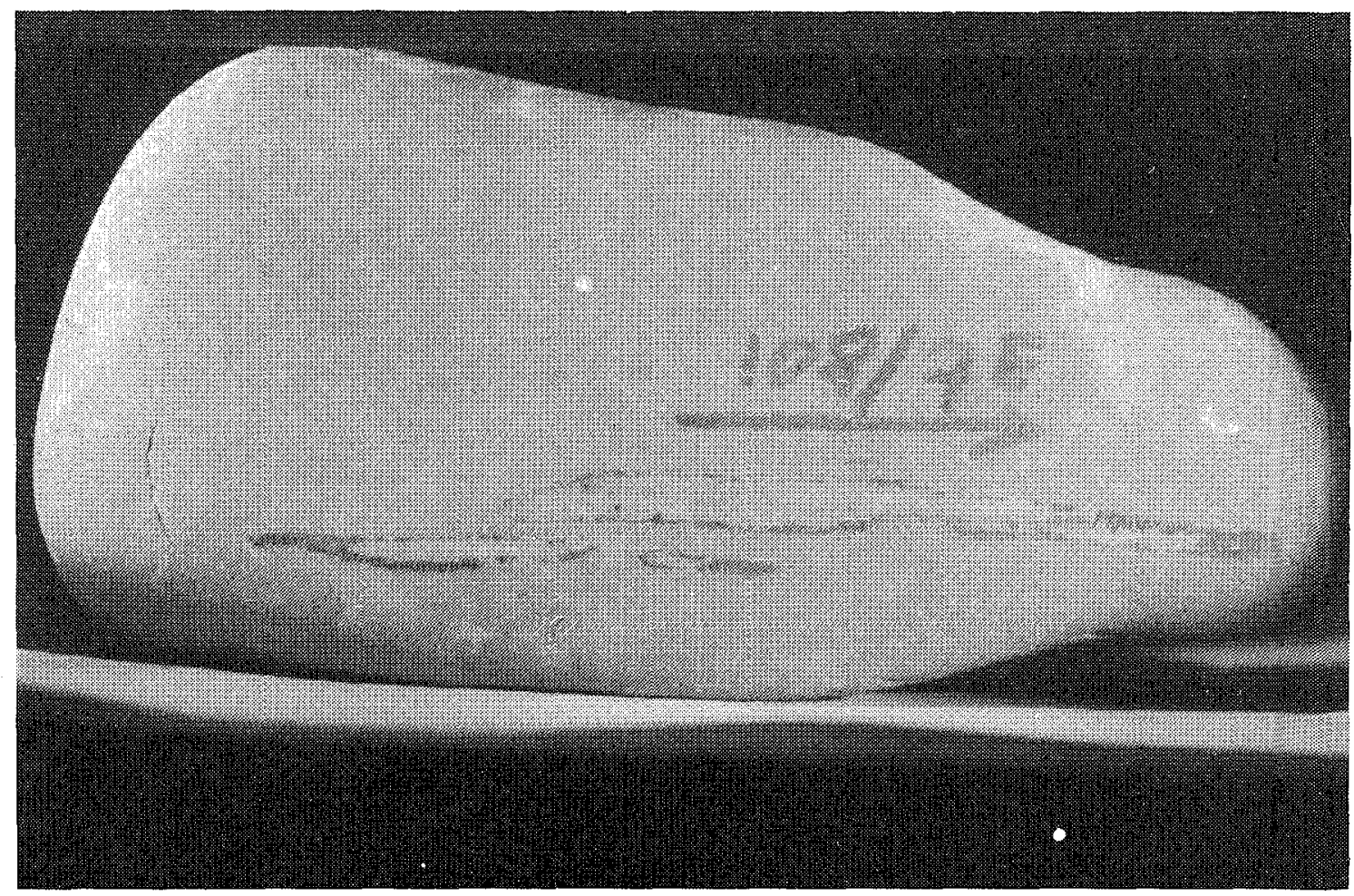

FOTO 4

Canto impreso por una estría. Talud de la Autopista del Ebro al este de Alcanadre. 


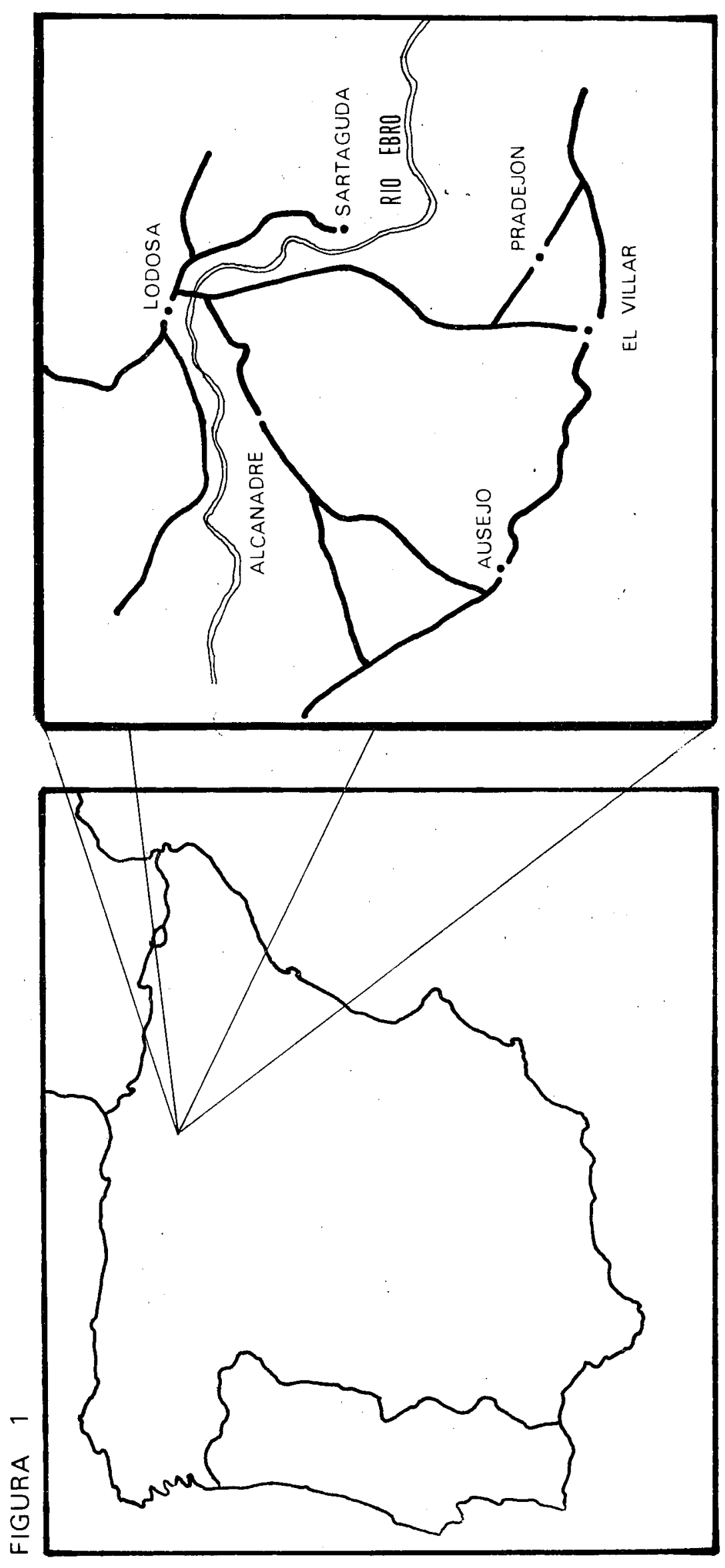


FALLAS CUATERNARIAS EN LA RIOJA BAJA

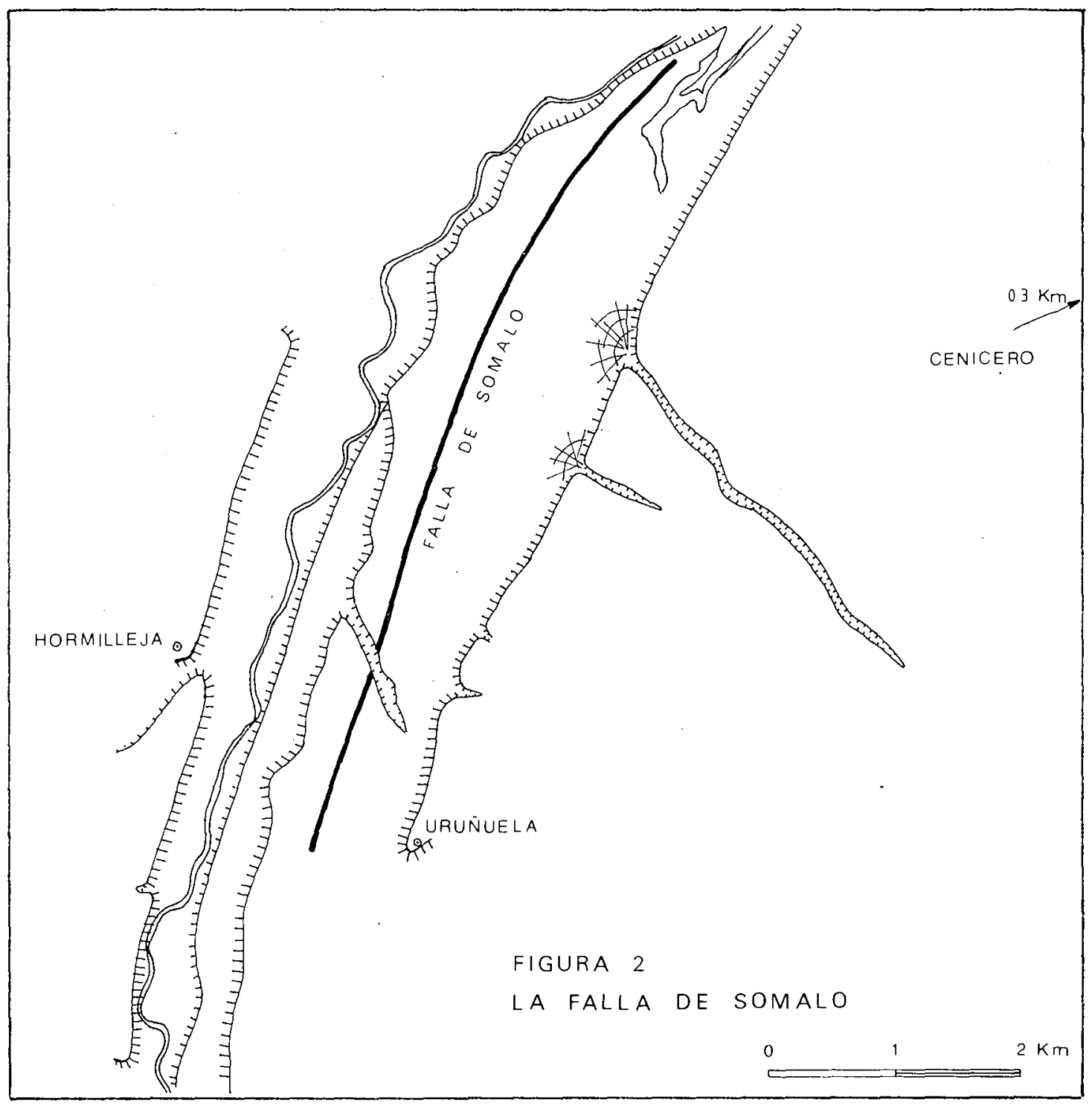




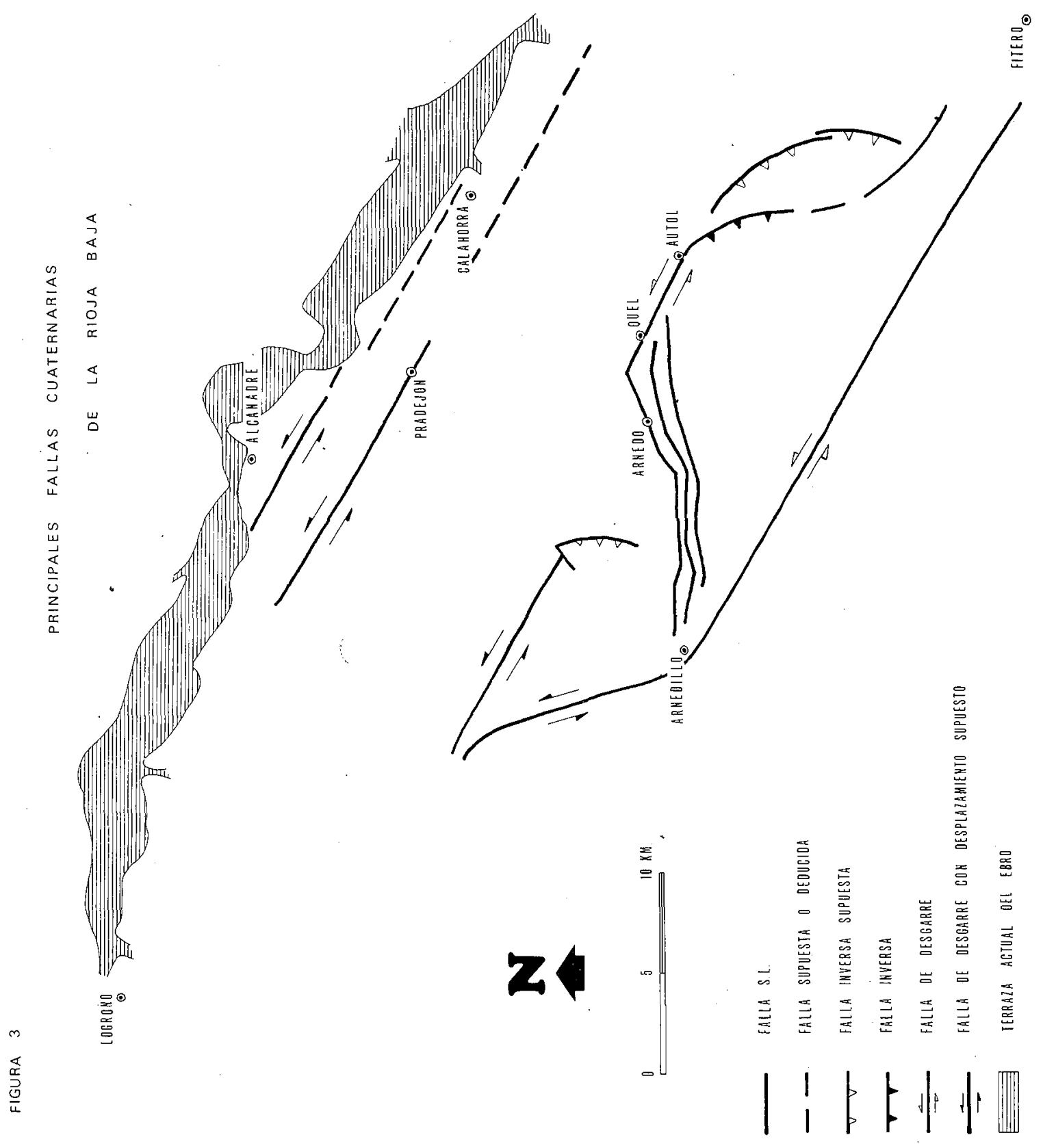


FALLAS CUATERNARIAS EN LA RIOJA BAJA

FIGURA 4

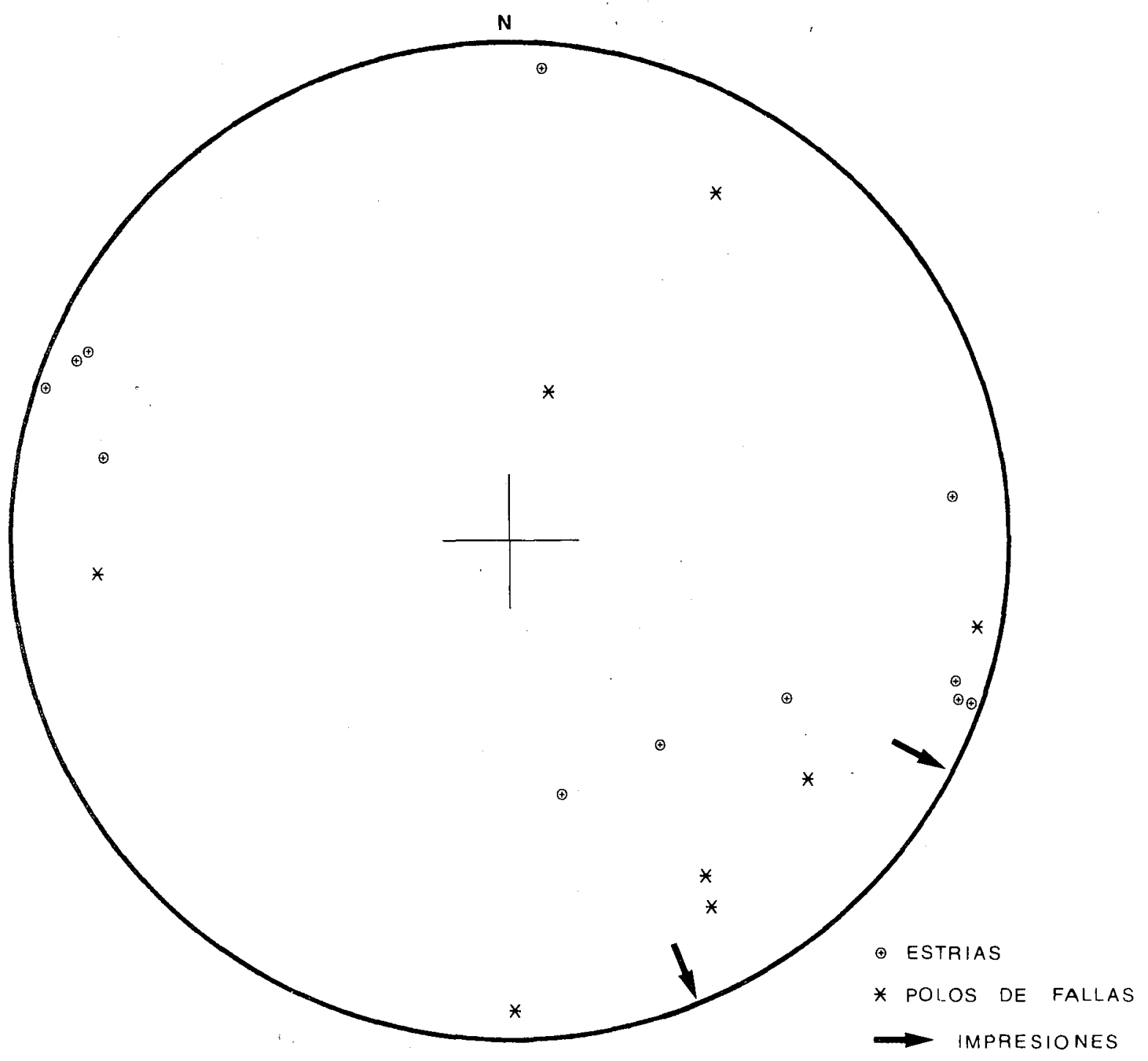

39 
